\title{
IDQMS: AN INTELLIGENT DATA QUALITY MANAGEMENT SYSTEM TOOL
}

\author{
Aïcha Ben Salem ${ }^{1,2}$ and Faouzi Boufares ${ }^{1}$ \\ ${ }^{1}$ LIPN, UMR CNRS 7030, Villetaneuse, France \\ ${ }^{2}$ RIADI, Manouba, Tunisia
}

\begin{abstract}
Today, the quantity of data continues to increase; furthermore, the data are distributed and heterogeneous, from multiple sources (structured, semi-structured and unstructured) and with different levels of quality. Therefore, it is very likely to manipulate data without knowledge about their structures and their semantics. The subject covered in this paper aims at assisting the user in its quality approach. The data must be related to its semantic meaning, data types, constraints, comments and origin. We deal with the semantic schema recognition of a data source. It consists of cate gorizing the data by assigning it to a category and possibly a sub-category, and secondly, of establishing relations between columns and possibly discovering the semantics of the manipulated data source. These links detected between columns offer a better understanding of the source and the alternatives for correcting data.
\end{abstract}

\section{KEYWORDS}

Data Quality, Semantic Data Categorization, Semantic Dependencies, Data Cleaning

\section{INTRODUCTION}

Data quality is fundamental to ensure intelligent knowledge mining from databases. Decision-making using erroneous data has a bad influence on the activities of organizations. Quantity of data continues to increase as well as the risks of anomalies (Boufarès et al-1, 2012). The automatic correction of these anomalies is a topic that is becoming more important both in business and in the academic world (Peralta, 2006), (Boufarès et al-1, 2012) (Boufarès et al-2, 2012), (Dallachiesay et al, 2013), (Ben Salem, 2015), (Chu et al, 2015), (Zaidi, 2017), (Zaidi et al, 2016). The data may be derived from different sources for which metadata can be absent and most often not sufficient to reflect the actual content of the data and treat any anomalies. Most ETL (Extract-Transform-Load tools) and MDM (Master data Management tools) do not assist the user during the integration and cleaning process. Therefore, it is interesting to create new data integration tools. Their role is to assist the user throughout the data integration and cleaning process. Our objective is then to assist to understand the semantics (Madnick et al, 2005) of the data in order to help detect and correct abnormalities.

The anomalies in the data were classified into several categories: (i) intra-column (data format, null value, outlier), (ii) inter-column (semantic dependencies between columns) and (iii) inter-line (duplicates and similar). Various problems could be faced by the user: (i) integration of heterogeneous data; (ii) different levels of data description: Little or no description at all; (iii) lack of semantics; (iv) variable quality. For instance, how can the following data (figure 2) be deciphered? Are there syntactic and semantic errors in the columns? Are there semantic links between columns (dependencies)? Is there consistency between the columns? Are there any semantic links between the lines (duplicates, similarities)? How can users discover these problems? and is there a way to discover and correct them automatically? How to detect and correct them automatically?

It should be noted that existing tools such as Talend ${ }^{1}$, Pentaho ETL ${ }^{2}$ and Oracle $\mathrm{MDM}^{3}$ do not take into account different information (e.g. meaning and semantics of the data, functional dependencies between columns), which help users in their profiling and cleaning process. No constraint detection process is used to

\footnotetext{
${ }^{1}$ https://www.talend.com/

2 https://www.pentaho.com/product/data-integration

${ }^{3}$ https://www.oracle.com
} 
identify anomalies. The user must be an expert or with minimum domain knowledge to firstly, understand the definition of the source data, and secondly, to decide the different conversions and transformations to be done on data. It is the user's responsibility to define the output model (the detailed structure of the data results). These tools are used to verify only the functional dependencies given by the user.

During an integration operation, ETLs take into account the compatibility between data types. The semantics of the column (column name and content) are not considered when integrating. In case of a join operation, the synonymy of column names is used to select the join key. But these names if they exist, do not represent the semantics defined in the data. Different issues may be generated due to a misinterpretation of the schema.

We propose an intelligent Data Quality Management System tool (iDQMS) which aims to better understand the data definition (semantic column name, data type and some constraints) and to better detect and correct anomalies. Figure 1 presents iDQMS tool architecture. As input it takes a data source and the data dictionaries and as output it provides some data reports and a cleaned data source.

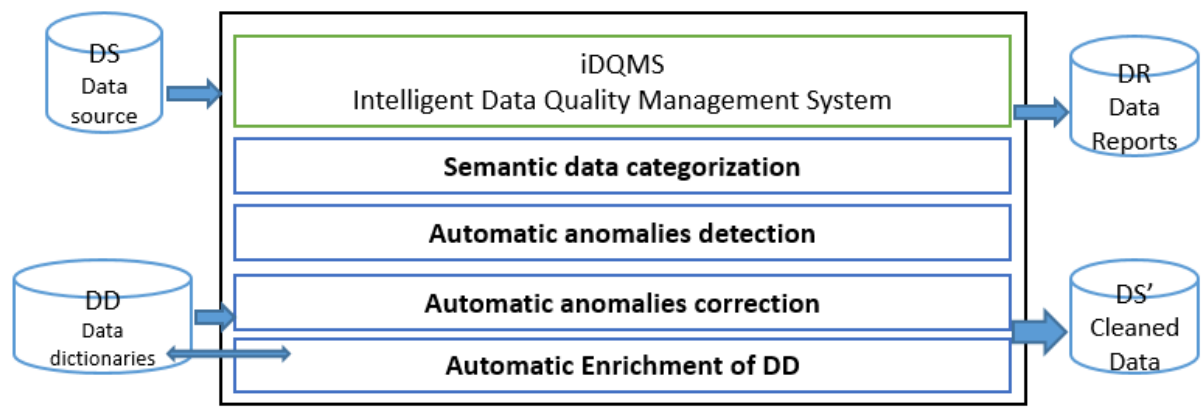

Figure 1. iDQMS tool architecture

In this paper, our proposal is to try to understand the semantics of the data before correcting it. We present in the section 2 the semantic data categorization (semantic columns name, syntactic data types, constraints, comments). In section 3, the intra and inter-columns semantic corrections are presented. We conclude with some perspectives.

\section{SEMANTIC DATA CATEGORIZATION}

Nowadays, a lot of data exchange is done with files in csv format. As a result, few metadata is available to integrate and exploit this data, let alone to detect possible anomalies.

Let us consider the above data source (figure 2), which is the result of the integration of two databases. The string "Pari" can be considered syntactically incorrect only if it is the French name of the city "Paris". The words "Pékin" and "Beijing" mean the same thing in two different languages if we know that these are names of cities. "Beijing" could be considered semantically incorrect if the used language (dominant language) is French; 2) The three strings "16-10-1996", "10-16-1996" and "1996-16-10" may represent the same type of information "date with different formats" defined by a regular expression; 3) The string "1996-10" is not a date.

There are currently no tools that bring the strings "Pékin" to "Beijing" or even "Londres" to "London". Additional semantic information is needed to know that these strings represent the same category "City" and the same subcategory "Language" of information. Similarly, it is important to recognize semantically the meaning of the string "sg@gmail.com" which is an email. 


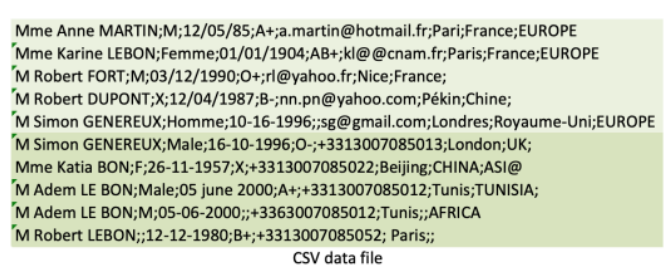

\begin{tabular}{|c|c|c|c|c|c|c|c|}
\hline Col1 & Col2 & Col3 & Col4 & Col5 & Col6 & Col7 & Col8 \\
\hline Mme Anne MARTIN & M & $12 / 05 / 85$ & At & a.martin@hotmail.fr & Pari & France & EUROPE \\
\hline Mme Karine LEBON & Femme & & $\mathrm{AB}+$ & k1@@cnam.fr & Paris & France & EUROPE \\
\hline M Robert FORT & M & $03 / 12 / 1990$ & $0+$ & rl@yahoo.fr & Nice & France & \\
\hline M Robert DUPONT & $\mathrm{x}$ & $12 / 04 / 1987$ & B- & nn.pn@yahoo.com & Pékin & Chine & \\
\hline M Simon GENEREUX & Homme & $10-16-1996$ & & sg@gmail.com & Londres & Royaume-Uni & EUROPE \\
\hline M Simon GENEREUX & Male & 16-10-1996 & $0-$ & +3313007085013 & London & UK & \\
\hline Mme Katia BON & $\mathrm{F}$ & 26-11-1957 & $x$ & +3313007085022 & Beijing & CHINA & ASI@ \\
\hline M Adem LE BON & Male & 05 june 2000 & At & +3313007085012 & Tunis & TUNISIA & \\
\hline M Adem LE BON & M & \begin{tabular}{|l|}
$05-06-2000$ \\
\end{tabular} & & +3363007085012 & Tunis & & AFRICA \\
\hline M Robert LEBON & & $12-12-1980$ & $B+$ & +3313007085052 & Paris & & \\
\hline
\end{tabular}

Figure 2. A sample of a data source DS

Let us consider DS as a set of tuples. Each tuple is composed of its $n$ columns. A column is defined by a domain $\operatorname{dom}\left(\operatorname{col}_{\mathrm{i}}\right)$ with $\mathrm{i}=1, \mathrm{n}$. An instance of a data source, defined on $\mathrm{n}$ columns, is a subset of the Cartesian product of the domains of these columns. $\mathrm{DS}=\left\{\mathrm{v}_{\mathrm{i}}, \mathrm{i}=1, \mathrm{x}\right\}$.

We can easily see that it is necessary to contextualize the data on the one hand and to find the data schema on the other hand. Our objective is then to build the metadata for the data source in csv format (column meaning, data type, constraints and comments).

We propose several data dictionaries (Ben Salem et al, 2014), (Ben Salem, 2015), (Zaidi, 2017) such as a data dictionary for valid strings DDVS and a data dictionary for regular expressions DDRE. They allow the categorization of data and help to detect and correct anomalies.

Data defined by extension: a priori given list such as city names, country names and names of organizations.

DDVS is defined as a set of tuples <Category, Sub-Categories, Others, Links between categories> (Figure 3).

DDVS $=\left\{w_{p}, p=1,1\right\}$ with $w_{p}$ is a valid string.

Note that this DDVS dictionary corresponds to a semantic network. Dependencies between categories are mentioned.

\begin{tabular}{|c|c|c|c|c|c|c|}
\hline Categories & \multicolumn{4}{|c|}{ Sub-Categories } & \multicolumn{2}{|c|}{ Links between categories } \\
\hline Category & English & French & Arabic & Others & Primary key & Foreign key \\
\hline \multirow{3}{*}{ CONTINENT } & AFRICA & AFRIQUE & أفريقيا & $\mathrm{AF}$ & Continent01 & \\
\hline & ASIA & ASIE & آسيا & AS & Continent 02 & \\
\hline & EUROPE & CITY & أوروبا & EU & Continent 03 & \\
\hline \multirow{3}{*}{ COUNTRY } & TUNISIA & TUNISIE & $\ldots$ & TUN & Country001 & Continent 01 \\
\hline & CHINA & CHINE & & $\mathrm{CN}$ & Country002 & Continent 02 \\
\hline & FRANCE & FRANCE & & FR & Country003 & Continent 03 \\
\hline \multirow{2}{*}{ CITY } & BEIJING & PEKIN & & & City001 & Country002 \\
\hline & PARIS & PARIS & & & City002 & Country003 \\
\hline \multirow{2}{*}{ FIRSTNAME } & AÏCHA & AÏCHA & & Female - Arab & & \\
\hline & AMELIE & AMELIE & & Female - Latin & & \\
\hline
\end{tabular}

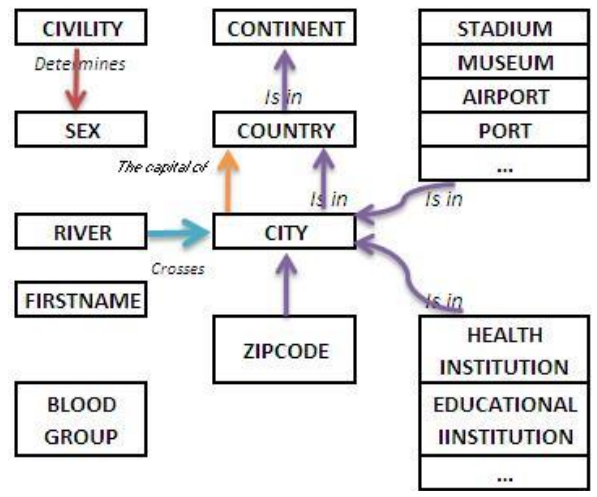

Semantic links between categories (DDVS+DDRE)

Figure 3. DDVS and Semantic links between categories

The semantic data recognition consists to find similarities between the DS and the valid strings in the DDVS to infer the semantic name of each column. We use measurements of similarity distance. We propose a mixture of methods of similarity measure, on the one hand, "it is written as", such as Jaro-Winkler (Koudas et al, 2006) or Levenshtein (Levenshtein, 1996), and on the other hand, "is pronounced as", such as Soundex (Koudas et al, 2006) or Metaphone (Koudas et al, 2006).

Data defined by intention: values must verify some properties such as regular expressions, for example Emails, Websites and Dates.

DDRE defined as a set of tuples <Category, Sub-Categories, Regular-expressions, Links between categories>

$\operatorname{DDRE}=\left\{\mathrm{e}_{\mathrm{j}}, \mathrm{q}=1, \mathrm{k}\right\}$ with $\mathrm{e}_{\mathrm{j}}$ is a regular expression. 
Table 1. An instance of data dictionary of regular expressions (DDRE)

\begin{tabular}{|c|c|c|c|}
\hline Category & SubCategory & RegEX & $\ldots$ \\
\hline \multirow{2}{*}{ TEMPERATURE } & TEMPERATURE_CELSIUS & [+-]?[0-9]+[[:space:]]?( $\left.\left.{ }^{\circ} \mathrm{C}\right|^{\circ} \mathrm{CELSIUS} \mid \mathrm{CELSIUS}\right) \$$ & \\
\hline & TEMPERATURE_FAHRENHEIT & {$[+-] ?[0-9]+[[:$ space: $]] ?\left({ }^{\circ} \mathrm{F}\left|{ }^{\circ} \mathrm{FAHRENHEIT}\right|\right.$ FAHRENHEIT $) \$$} & \\
\hline \multirow{2}{*}{ WEIGHT } & WEIGHT_KG & [0-9]+[[:space:]]?(KG|KILOGRAM|KILOGRAMS)\$ & \\
\hline & WEIGHT_TO & {$[0-9]+[[$ :space:]]?(TO|TONNES|TONNE)\$ } & \\
\hline BLOODGROUP & BLOODGROUP & $\mathrm{A}+|\mathrm{A}-| \mathrm{B}+|\mathrm{B}-| \mathrm{AB}+|\mathrm{AB}-| \mathrm{O}+\mid \mathrm{O}-$ & \\
\hline \multirow{2}{*}{ DATE } & DATE-FR-DD-MM-AAAA & {$[0-3] ?[0-9][\backslash-\backslash].(0 ?[1-9]|[12][0-9]| 3[01])[\backslash-\backslash.] \backslash \mathrm{d}\{4\} \$$} & \\
\hline & DATE-USA-MM-DD-AAAA & $([12] \backslash d\{3\}-(0[1-9] \mid 1[0-2])-(0[1-9]|[12] \backslash d| 3[01]))$ & \\
\hline EMAIL & EMAIL & $\wedge\left[\mathrm{A}-\mathrm{Za}-\mathrm{z} 0-9 . \_\%-\right]+@[\mathrm{~A}-\mathrm{Za}-\mathrm{z} 0-9 .-]+\backslash .[\mathrm{A}-\mathrm{Za}-\mathrm{z}]\{2,4\} \$$ & 四 \\
\hline
\end{tabular}

We propose other types of dictionaries that make it possible to define the syntactic type of each category, to mention some constraints necessary to detect some anomalies. These dictionaries contain, for example, the list of categories (DDCAT), the list of constraints (DDConstraints), the list of semantic links (DDLinks).

DDCAT as a set of tuples <DicType, Category, Constraints, SyntacticType, MinLength, MaxLength, Similarity algorithm, Threshold, ...>.

Table 2. An instance of the dictionary of categories

\begin{tabular}{|l|l|l|c|c|c|c|c|}
\hline DicType & \multicolumn{1}{|c|}{ Category } & Constraints & $\begin{array}{c}\text { Syntactic } \\
\text { Type }\end{array}$ & $\begin{array}{l}\text { Min } \\
\text { Length }\end{array}$ & $\begin{array}{c}\text { Max } \\
\text { Length }\end{array}$ & Similarity algorithm & Threshold \\
\hline DDVS & CONTINENT & & String & 4 & 7 & Edit-Distance & 2 \\
\hline DDVS & COUNTRY & Country $\rightarrow$ Continent & String & 4 & 32 & Edit-Distance & 2 \\
\hline DDVS & CITY & City $\rightarrow$ Country & String & 2 & & Edit-Distance & 2 \\
\hline DDVS & FIRST NAME & & String & 2 & & Jaro-Winkler & 2 \\
\hline DDRE & EMAIL & & String & & & Soundex & 0 \\
\hline DDRE & BLOODGROUP & $\epsilon\{\mathrm{A}+, \mathrm{B}+, \mathrm{AB}+, \mathrm{O}+, \ldots\}$ & String & 2 & 3 & Edit-Distance & 0 \\
\hline DDRE & CREDITCARDNUMBER & & String Numeric & 16 & 16 & Edit-Distance & 0 \\
\hline DDRE & ZIPCODE & ZipCode $\rightarrow$ City & String & 2 & 9 & Q-Gram & 0 \\
\hline
\end{tabular}

After defining our dictionaries let us, present our semantic data structure process.

\section{Semantic data categorization process}

The categorization process consists of going through all the values of a column and deducting the corresponding category from it through the DDVS and DDRE dictionaries.

Table 3. DS initial schema

\begin{tabular}{|c|c|c|c|c|c|c|c|c|c|}
\hline & \multicolumn{9}{|c|}{ Old Data Schema: Starting schema of the data source } \\
\hline \multirow{4}{*}{ 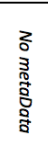 } & Colunm & Col1 & $\mathrm{Col} 2$ & $\mathrm{Col} 3$ & Col4 & Col5 & Col6 & Col7 & Col8 \\
\hline & DataType & String & String & String & String & String & String & String & String \\
\hline & Constraint & & & & & & & & \\
\hline & Comments & & & & & & & & \\
\hline
\end{tabular}

Let DS $\left(\mathrm{Col}_{1}, \mathrm{Co}_{12}, \ldots, \mathrm{Col}_{\mathrm{n}}\right)($ table 3$)$ with $\mathrm{Col}_{\mathrm{i}}=\left\{\mathrm{v}_{\mathrm{i}}, \mathrm{i}=1, \mathrm{~m}\right\}$.

Definition 1 (Valid value $\mathbf{v}$ ): $\mathrm{v}$ is valid iff $\exists \mathrm{e} \in$ DDRE where $\mathrm{v} \mathcal{E}$ e or $\exists \mathrm{w} \in$ DDVS where $\mathrm{v} \simeq \mathrm{w}$. $\mathrm{v}$ is an invalid value otherwise.

Where $\mathrm{v} E$ e means that a value $\mathrm{v}$ verifies a regular expression $\mathrm{e} ; \mathrm{v} \simeq \mathrm{w}$ means that similarityDistance $(\mathrm{w}, \mathrm{v}) \leq$ threshold. 
The iDQMS tool allows you to create a set of data reports (DR) such as data indicators, possible indications and corrections of invalid values or semantic links between columns.

Definition 2 (Data indicator): $\mathrm{M}_{\alpha}=\mathrm{f}$ (file-table, column) where $\mathrm{f}$ is a function that performs statistical analyses on the data (such as the number of missing values or the number of invalid values)

Data cleaning should start with a diagnostic of anomalies. This action is commonly referred to as data profiling. This consists of calculating indicators (Ben Salem, 2015), (Zaidi, 2017) (table 4), such as: total number of tuples in the DS $\left(\mathrm{M}^{0}{ }_{00}\right)$; total number of columns in the DS $\left(\mathbf{M}^{0}{ }_{01}\right)$; number of distinct values in the column $\left(\mathrm{M}^{1}{ }_{01}\right)$; number of null values in the column $\left(\mathrm{M}^{1}{ }_{02}\right)$; number of invalid values in the column $\left(\mathrm{M}^{1}{ }_{03}\right)$; number of categories per column $\left(\mathrm{M}^{1}{ }_{04}\right)$; number of subcategories per column $\left(\mathrm{M}^{1}{ }_{05}\right)$; the minimum length of strings per column $\left(\mathrm{M}^{1}{ }_{06}\right)$; the maximum length of strings per column $\left(\mathrm{M}^{1}{ }_{07}\right)$; the number of words to be considered in the same column $\left(\mathrm{M}^{1}{ }_{08}\right)$; semantic relationship (=) between two columns $\left(\mathrm{M}^{2}{ }_{01}\right)$; semantic relationship $(<)$ between two columns $\left(\mathrm{M}^{2}{ }_{02}\right)$; semantic relationship $(>)$ between two columns $\left(\mathrm{M}^{2}{ }_{03}\right)$; functional dependencies $(\rightarrow)$ between two columns $\left(\mathrm{M}^{2}{ }_{04}\right)$.

Table 4. Indicators measurement applied to DS

\begin{tabular}{|c|c|c|c|c|c|c|c|c|c|c|c|c|}
\hline & $\mathrm{M}_{01}^{1}$ & $\mathrm{M}_{02}^{1}$ & $\mathrm{M}_{03}^{1}$ & $\mathrm{M}_{04}^{1}$ & $\mathrm{M}_{05}^{1}$ & $\mathrm{M}_{06}^{1}$ & $\mathrm{M}_{07}^{1}$ & $\mathrm{M}_{08}^{1}$ & $\mathrm{M}_{01}^{2}$ & $\mathrm{M}_{02}^{2}$ & $\mathrm{M}_{03}^{2}$ & $\mathrm{M}_{04}^{2}$ \\
\hline Col1 & 8 & 0 & 0 & 0 & 0 & 13 & 16 & 4 & - & - & - & - \\
\hline Col2 & 2 & 0 & 1 & 1 & 0 & 1 & 1 & 1 & - & - & - & - \\
\hline Col3 & 8 & 1 & 1 & 1 & 1 & 8 & 8 & 1 & - & - & - & - \\
\hline Col4 & 6 & 2 & 1 & 1 & 0 & 2 & 3 & 1 & - & - & - & - \\
\hline Col5 & 10 & 0 & 1 & 2 & 0 & 11 & 19 & 1 & - & - & - & - \\
\hline Col6 & 8 & 0 & 0 & 2 & 2 & 7 & 11 & 1 & - & - & - & $\rightarrow$ Col7 \\
\hline Col7 & 6 & 2 & 2 & 2 & 2 & 2 & 11 & 1 & - & - & - & $\rightarrow$ Col8 \\
\hline Col8 & 4 & 5 & 1 & 2 & 2 & 3 & 6 & 1 & - & - & - & - \\
\hline
\end{tabular}

The indicators make it possible to detect several cases of restructuring of the source scheme. For instance, columns 1 and 6 can be split into several columns, as column 1 contains mainly 3 words and column 6 corresponds to two categories. The indicators we propose can give an idea of future actions that could be taken on a column, such as grouping attributes or duplicate attributes.

Definition 3 (Grouping Attribute): A column is considered as a grouping attribute if $\mathrm{M}^{1}{ }_{02} / \mathrm{M}^{0}{ }_{00} \leq \beta_{1}$ and $\mathrm{M}^{1}{ }_{01} / \mathrm{M}^{0}{ }_{00} \simeq \beta_{2}\left(\beta_{2} \simeq 0\right)$. The latter would allow subtotals to be established such as Min, Max, Average or Sum.

Definition 4 (Duplicate Attribute): A column is considered as a duplicate attribute if $\mathrm{M}^{1}{ }_{02} / \mathrm{M}^{0}{ }_{00} \leq \beta_{1}$ and $\mathrm{M}^{1}{ }_{01} / \mathrm{M}^{0}{ }_{00} \simeq \beta_{2}\left(\beta_{2} \simeq 1\right)$.

The categorization process, which we propose, enables us to assist in data management: restructuring the data source, semantically enriching the metadata, helping in the choice of deduplication attributes and similarity calculation algorithms (table 5).

Table 5. New semantic data schemas

\begin{tabular}{|c|c|c|c|c|c|c|c|c|c|c|c|c|}
\hline & \multicolumn{12}{|c|}{ New Data Schema (SCH1): Schema from the diagnostic step } \\
\hline \multirow{11}{*}{ 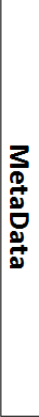 } & Initial Colunm Name & Col11 & Col12 & Col13 & Col2 & Col4 & Col5 & Col61 & Col62 & Col4 & Col7 & Col8 \\
\hline & Sub-category (Semantic) & French & & & French & & & & French & French & French & French \\
\hline & \multirow{3}{*}{ Constraints } & M.|Mme & $[A-Z]\{1\}][a-z]+$ & & $\mathrm{M} \mid \mathrm{F}$ & YYYY-MM-DD & & & & RegEX: $[A-Z]$ & RegEX: $[A-Z]$ & RegEX: $[A-Z]$ \\
\hline & & & \multicolumn{2}{|c|}{ Prohibition of 3 consecutive letters } & & & & & & \multicolumn{3}{|c|}{\begin{tabular}{|c|} 
Prohibition of 3 consecutive letters \\
\end{tabular}} \\
\hline & & Civility $\rightarrow$ Gender & & & & & & & & City $\rightarrow$ Country & \multicolumn{2}{|c|}{ Country $\rightarrow$ Continent } \\
\hline & Comments & List of civilities & List of ... & & List of ... & A date form & & List of ... & List of ... & List of ... & List of ... & List of ... \\
\hline & Min Length & 1 & 2 & & 1 & & 2 & & & 2 & 2 & 4 \\
\hline & Max Length & & & & & & 3 & & & & & 7 \\
\hline & Grouping Attribute & * & & & * & * & * & & & * & * & \\
\hline & Duplicate Attribute & & * & & & & & ** & ** & * & & \\
\hline & Semantic Links $(<,=,>, \rightarrow)$ & $\rightarrow$ Gender $(99 \%)$ & & & & & & & & $\rightarrow$ Country $(80 \%)$ & $\mid \rightarrow$ Continent $(90 \%)$ & \\
\hline
\end{tabular}


Intermediate reports returned by the semantic data categorization process, can detect the presence of certain anomalies in the data source such as non-standard data and null values.

\section{DATA CLEANING}

Data cleaning consists on correction of intra and inter-columns anomalies. For that, we must explicit links between columns. Constraint checking dependencies from large amounts of data will help to enrich automatically the data source.

\subsection{Homogenization}

The semantic knowledge deduced from the step of semantic categorization allows correcting intra-column anomalies. We call this phase homogenization/standardization of data. The syntactical correction of data is done by approximating the values of the data source to those, which are similar in the Data Dictionary. We use methods of similarity distance calculation. Our cleaning process can correct misspelled values. It allows standardizing formats. We propose also to unify the data in the same subcategory. Then, values that do not belong to the dominant subcategory are translated into their synonyms (figure 4).

\begin{tabular}{|c|c|c|c|}
\hline \multirow{4}{*}{$\begin{array}{c}\text { Syntax } \\
\text { corrections }\end{array}$} & Category & Old values & New values \\
\cline { 2 - 4 } & City & Pari & PARIS \\
\cline { 2 - 4 } & City & Pikin & PÉKIN \\
\hline \multirow{2}{*}{$\begin{array}{c}\text { Semantic } \\
\text { corrections }\end{array}$} & City & Beijing & PÉKIN \\
\cline { 2 - 4 } & Country & Tunisia & TUNISIE \\
\hline \multirow{4}{*}{ Unification } & Gender & Homme & M \\
\cline { 2 - 4 } & Gender & Femme & F \\
\cline { 2 - 4 } & Col4_Date & 05 june 2000 & $05 / 06 / 2000$ \\
\cline { 2 - 4 } & Col4_Date & $12 / 05 / 85$ & $12 / 05 / 1985$ \\
\hline
\end{tabular}

Examples of intra-column automatic transformations

\begin{tabular}{|c|c|c|c|c|c|c|c|c|c|c|}
\hline Civility & FirstName & Unknown & Gender & Col4_Date & BloodGroup & Email & Phone & City & Country & Continent \\
\hline Mme & Anne & MARTIN & M & $12 / 05 / 1985$ & $\mathrm{~A}^{+}$ & a.martin@hotmail.fr & & PARIS & FRANCE & EUROPE \\
\hline Mme & Karine & LEBON & $\mathrm{F}$ & NULL & $\mathrm{AB}^{+}$ & k1@@enam.fr & & PARIS & FRANCE & EUROPE \\
\hline M & Robert & FORT & M & $03 / 12 / 1990$ & $\mathrm{O}^{+}$ & rl@yahoo.fr & & NICE & FRANCE & NULL \\
\hline M & Robert & DUPONT & $\mathrm{x}$ & $12 / 04 / 1987$ & B- & nn.pn@yahoo.com & & PÉKIN & CHINE & NULL \\
\hline M & Simon & GENEREUX & M & $10-16-1996$ & NULL & sg@gmail.com & & LONDRES & ROYAUME-UNI & EUROPE \\
\hline $\mathrm{M}$ & Simon & GENEREUX & M & $16 / 10 / 1996$ & $0-$ & & +3313007085013 & LONDRES & ROYAUME-UNI & NULL \\
\hline Mme & Katia & BON & F & $26 / 11 / 1957$ & $\mathrm{x}$ & & +3313007085022 & PÉKIN & CHINE & ASIE \\
\hline M & Adem & LE BON & M & $05 / 06 / 2000$ & $\mathrm{~A}^{+}$ & & +3313007085012 & TUNIS & TUNISIE & NULL \\
\hline $\mathrm{M}$ & Adem & LE BON & M & $05 / 06 / 2000$ & NULL & & +3363007085012 & TUNIS & NULL & AFRIQUE \\
\hline M & Robert & LEBON & NULL & $12 / 12 / 1980$ & $\mathrm{~B}^{+}$ & & +3313007085052 & PARIS & FRANCE & EUROPE \\
\hline
\end{tabular}

Intra-column automatic corrections done on the DS

Figure 4. Data source homogenization (DS')

We still have to correct the colored cells (contradictions, an outlier for the month, incorrect values and null values). 


\subsection{Data Enrichment}

The correction of intra-column anomalies facilitates the verification of semantic links between the columns. The DDCAT dictionary allows inferring the different semantic links such as the functional dependencies (Dong et al, 2009), (Simonenko et al, 2012), (Zaidi, 2017) that may exist between categories. It is necessary to deduce all other links based on the transition of functional dependencies: If $X \rightarrow Y$ and $Y \rightarrow Z$ Then $X \rightarrow Z$. Indeed, in a data source, not all categories are necessarily present. Thus, the basic functional dependencies are not sufficient to deduce all possible links in the data source. DDCAT can be seen as calculated semantic links and stored in DDLINKS (table 6) by performing self-joints.

Table 6. An instance of the DDLINKS

\begin{tabular}{|l|l|}
\hline \multicolumn{2}{|c|}{ DEPENDENCIES $:$ Left $\rightarrow$ Right } \\
\hline CATEGORY Left & CATEGORY Right \\
\hline CITY & COUNTRY \\
\hline CITY & CONTINENT \\
\hline COUNTRY & CONTINENT \\
\hline CIVILITY & GENDER \\
\hline
\end{tabular}

Our approach is to enrich the data source and to fill in some null values using the semantic dependencies between columns.

We use the valid values stored in the DDVS. Some null values are then processed and corrected according to deductions extracted from the data (table 7). Let us note that the order of treatment of functional dependencies is very important to complete null values. It is necessary to start with the columns that are less empty.

Table 7. Inter-Columns automatic corrections done on the DS (DS')

\begin{tabular}{|c|c|c|c|c|c|c|c|c|c|c|}
\hline Civility & FirstName & Unknown & Gender & Col4_Date & BloodGroup & Email & Phone & City & Country & Continent \\
\hline Mme & Anne & MARTIN & M & $12 / 05 / 1985$ & $\mathrm{~A}^{+}$ & a.martin @ hotmail.fr & & PARIS & FRANCE & EUROPE \\
\hline Mme & Karine & LEBON & F & NULL & $\mathrm{AB}+$ & kl@@ cnam.fr & & PARIS & FRANCE & EUROPE \\
\hline M & Robert & FORT & M & $03 / 12 / 1990$ & $\mathrm{O}+$ & rlayahoo.fr & & NICE & FRANCE & EUROPE \\
\hline M & Robert & DUPONT & M & $12 / 04 / 1987$ & B- & nn.pn@yahoo.com & & PÉKIN & CHINE & ASIE \\
\hline M & Simon & GENEREUX & M & 10-16-1996 & NULL & sg@gmail.com & & LONDRES & ROYAUME-UNI & EUROPE \\
\hline M & Simon & GENEREUX & M & $16 / 10 / 1996$ & o. & & +3313007085013 & LONDRES & ROYAUME-UNI & EUROPE \\
\hline M & Adem & LE BON & M & $05 / 06 / 2000$ & $\mathrm{~A}^{+}$ & & +3313007085012 & TUNIS & TUNISIE & AFRIQUE \\
\hline M & Adem & LE BON & M & $05 / 06 / 2000$ & NULL & & +3363007085012 & TUNIS & TUNISIE & AFRIQUE \\
\hline M & Robert & LEBON & M & $12 / 12 / 1980$ & $\mathrm{~B}^{+}$ & & +3313007085052 & PARIS & FRANCE & EUROPE \\
\hline
\end{tabular}

Currently the majority of ETL tools, for record linkage algorithms, require some expertise in data management. While our iDQMS tool recommends the necessary features for deduplication (using the definitions given above).

\section{CONCLUSION AND PERSPECTIVES}

The iDQMS tool offers the possibility to assist the user in the data quality process. Our contribution is to understand the semantic of data before correcting them. In fact, iDQMS tool enables the categorization of data by giving them a category, eventually a subcategory and different constraints. Semantic categorization allows inferring semantic links that can exist between the different columns. The recognition of the structure and semantics of data facilitates the detection and correction of various intra- and inter-columns anomalies in the same data source. Data categorization also detects and corrects duplicates, helps to select deduplication attributes based on their semantics and defines the corresponding similarity measure and threshold.

Among our future work, we plan to apply the basic concepts of machine learning in data management. This will make it easier to detect constraints from the data.

We propose the inference on ontologies for the recommendation of indicators to be applied on a column. 


\section{REFERENCES}

Ben Salem, A., 2015. Qualité contextuelle des données : Détection et nettoyage guidés par la sémantique des données, Ph.D. Thesis, Université Paris 13, Sorbonne Paris cité, Paris, France.

Ben Salem, A., Boufarès, F. and Correia, S., 2014. Semantic Recognition of a Data Structure in Big-Data. Proceedings of the 6th International Conference on Computational Intelligence and software Engineering, Vol. 2, Beijing, China, pp. 93-103.

Boufarès, F. and Ben-Salem. A., 2012. Heterogeneous data-integration and data quality: Overview of conflicts. In 6th International Conference on Sciences of Electronics, Technologies of Information and Telecommunications (SETIT'12), ISBN 978-1- 4673-1657-6, Sousse, Tunisie, pp. 867-874.

Boufarès, F. and Ben-Salem. A. and Correia. S., 2012. Qualité de données dans les entrepôts de données : élimination des similaires. In 8èmes Journées francophones sur les Entrepôts de Données et l'Analyse en ligne (EDA'12), RNTI-B 2012, Bordeaux, France, pp. 32-41.

Chu, X., Morcos, J., Ilyas, I.F, Ouzzani, M., Papotti, P., Tang, N. and Ye, Y., 2015. KATARA: A Data Cleaning System Powered by Knowledge Bases and Crowdsourcing, Proceedings of the 2015 ACM SIGMOD International Conference on Management of Data, Melbourne, Autralia, pp. 1247-1261.

Dallachiesay, M., Ebaidz, A., Eldawy, A., Elmagarmid, A., Ilyas, I.F., Ouzzani, M. and Tang, N., 2013. NADEEF: A Commodity Data Cleaning System, Proceedings of ACM SIGMOD International Conference on Management of Data, IEEE Press, New York, USA, pp. 541-552.

Dong. X.L., Berti-Equille. L., and Srivastava. D., 2009. Integrating conflicting data: the role of source dependence. In the 35th International Conference on Very Large Databases (VLDB '09), Lyon, France, pp. 550-561.

Koudas. N., Sarawagi. S., and Srivastava. D., 2006. Record Linkage: Similarity Measures and Algorithms. In the ACM International Conference on Management of Data (SIGMOD’06), Chicago, Illinois, USA, pp. 802-803.

Levenshtein. V.I., 1966, Binary Codes Capable of Correcting Deletions, Insertions and Reversals. In Doklady Akademii Nauk SSSR, vol. 163, no. 4, pp. 845-848.

Madnick. S. and Zhu. H., 2005. Improving data quality through effective use of data semantics. In Working paper CISL\#2005-08, pp 1-19.

Peralta. V., 2006, Data quality evaluation in data integration systems. PhD thesis, Versailles, France.

Simonenko. E. and Novelli. N., 2012. Extration de dépendances fonctionelles approximtives : une approche incrémentale. In Extractions et Gestions des Connaissances, RNTT E.23 (EGC'12), Bordeaux, France, pp. 95-100.

Zaidi, H., 2017. Amélioration de la qualité des données. Correction sémantique des anomalies inter-colonnes. Ph.D. Thesis, Conservatoire National des Arts et Métiers, Paris, France.

Zaidi, H., Boufarès, F. and Pollet, Y., 2016. Nettoyage de données guidé par la sémantique inter-colonne, Proceedings: 16th Conférence Internationale sur l'Extraction et la Gestion des Connaissances, Vol. RNTI-E-30, Reims, France, pp. 549-550. 